\title{
Nanoparticle-assisted acceleration of laser-irradiated low-density He ions
}

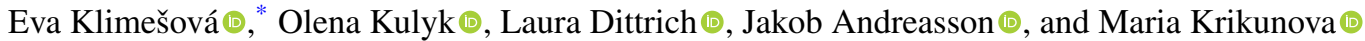 \\ ELI Beamlines, Institute of Physics AS CR, v.v.i., Na Slovance 2, 18221 Prague 8, Czech Republic
}

(Received 23 June 2021; accepted 8 November 2021; published 27 December 2021)

\begin{abstract}
We report on a surprisingly efficient acceleration of ambient gas ions in the presence of a laser-irradiated nanoparticle. Near-infrared laser pulses with an intensity of $2 \times 10^{15} \mathrm{~W} \mathrm{~cm}^{-2}$ are used to irradiate a single $\mathrm{CsCl}$ nanoparticle surrounded by a helium gas with a density of around $10^{15} \mathrm{~cm}^{-3}$. In this interaction $\mathrm{He}^{+}$ions gain higher maximum kinetic energy than $\mathrm{Cs}^{+}$and $\mathrm{Cl}^{+}$ions. We suggest that $\mathrm{He}^{+}$ions are accelerated by a positive charge at the nanoparticle surface whereas the movement of $\mathrm{Cs}^{+}$and $\mathrm{Cl}^{+}$ions is interlinked to the expansion of the nanoplasma electrons. Our results reveal an essential contribution of the environment to the interaction of intense light with dense matter.
\end{abstract}

DOI: 10.1103/PhysRevA.104.L061101

The interaction of nanoscale targets (with sizes from 10 to several $100 \mathrm{~nm}$ ) with intense near-infrared (NIR) laser pulses has remained in the focus of extensive research for several decades. Fascinating observations of unexpectedly high ionic charge states [1], ions with megaelectronvolt energies [2,3], energetic electrons [4,5], and x-ray radiation [6,7] reveal highly efficient absorption of the laser pulse energy. Novel phenomena, e.g., creation of shock waves in nanoplasmas [8], controlled electron emission from $\mathrm{SiO}_{2}$ nanoparticles [9-11], mapping laser absorption by ion emission measurement [12] or accelerating electrons in nanoplasmonic resonant systems [13], have been observed in laser-irradiated nanoparticles. Attractive application areas for laser-irradiated clusters and nanoparticles include generation of high-order harmonics [14-16] or the development of tabletop laser-electron accelerators where laser-irradiated nanoparticles should serve as a localized and controllable source of electrons for injection $[17,18]$.

To understand the complex interaction dynamics of nanoparticles with intense laser light an idealized case of a single isolated nanoparticle in vacuum is commonly considered. Little attention has been paid to the environment surrounding the nanoparticle. This picture might be incomplete since a substantial amount of uncondensed gas can be present in supersonic cluster beams as seen in high-resolution photoelectron spectra $[19,20]$. The ionization dynamics of clusters might remain hidden in the experiment due to the presence of an uncondenced gas in the cluster beam [21]. Furthermore, a laser-irradiated nanoparticle can considerably influence the dynamics of its environment as well. For instance, model calculations predict a significant enhancement of the kinetic-energy cutoff in atomic photoelectron spectra if

\footnotetext{
*eva.klimesova@eli-beams.eu

Published by the American Physical Society under the terms of the Creative Commons Attribution 4.0 International license. Further distribution of this work must maintain attribution to the author(s) and the published article's title, journal citation, and DOI.
}

atoms are ionized in a local plasmonic field in the vicinity of a nanoparticle surface [22]. The interaction of intense lasers with dense matter and its environment is actively explored for the optimization of laser-driven proton acceleration. In the target normal sheath acceleration scheme a laser-irradiated thin foil is commonly used as a target [23-25]. Different modifications to the surface of the foil have been investigated recently in order to enhance the laser energy absorption, e.g., creation of a preplasma by the target ablation [26,27], covering the surface of the foil with a monolayer of nanoparticles [28] or by purging the solid target with an atomic gas [29].

In this Letter we investigate how the acceleration dynamics of an underdense plasma is influenced by the presence of a single laser-irradiated nanoparticle. In our study a single $\mathrm{CsCl}$ nanoparticle (of $\sim 200$-nm diameter) is placed into a He gas of $5 \times 10^{15} \mathrm{~cm}^{-3}$ density and irradiated by an intense femtosecond NIR laser pulse. We use ion time-of-flight (ToF) mass spectrometry to discern between ionic fragments originating from the nanoparticle (mainly $\mathrm{Cs}^{+}, \mathrm{Cl}^{+}$, and $\mathrm{H}^{+}$ coming from water in the nanoparticle) and $\mathrm{He}^{+}$ions from the gas environment. This species-selective study allows us to unambiguously disentangle key mechanisms of ion acceleration: (i) nanoparticle-independent $\mathrm{He}^{+}$acceleration driven by Coulomb repulsion of laser-created plasma channel [30], (ii) expansion of nanoparticle ions $\left(\mathrm{Cs}^{+}, \mathrm{Cl}^{+}\right.$, and $\left.\mathrm{H}^{+}\right)$driven mainly by hydrodynamic forces [31], and (iii) nanoparticleassisted $\mathrm{He}^{+}$acceleration, which we attribute to the electric field on the nanoparticle created by fast escaping electrons.

The experiment was performed at ELI Beamlines in the Czech Republic at the MAC end station [32]. CsCl nanoparticles with a diameter around $200 \mathrm{~nm}$ were injected into a vacuum chamber through the "Uppsala injector" $[33,34]$. The injector consisted of a gas dynamic virtual nozzle (GDVN) [35] producing an aerosol from $0.1 \% \mathrm{CsCl}$ solution in water. Aerosol droplets were then delivered to the vacuum chamber via an aerodynamic lens stack (ALS) [36]. Water from the droplets evaporated on the way to the ALS exit leaving intact $\mathrm{CsCl}$ nanoparticles covered with a water layer [34,37] in the interaction region. In the process of guiding and focusing the nanoparticle beam, helium was used as the carrier gas, having 

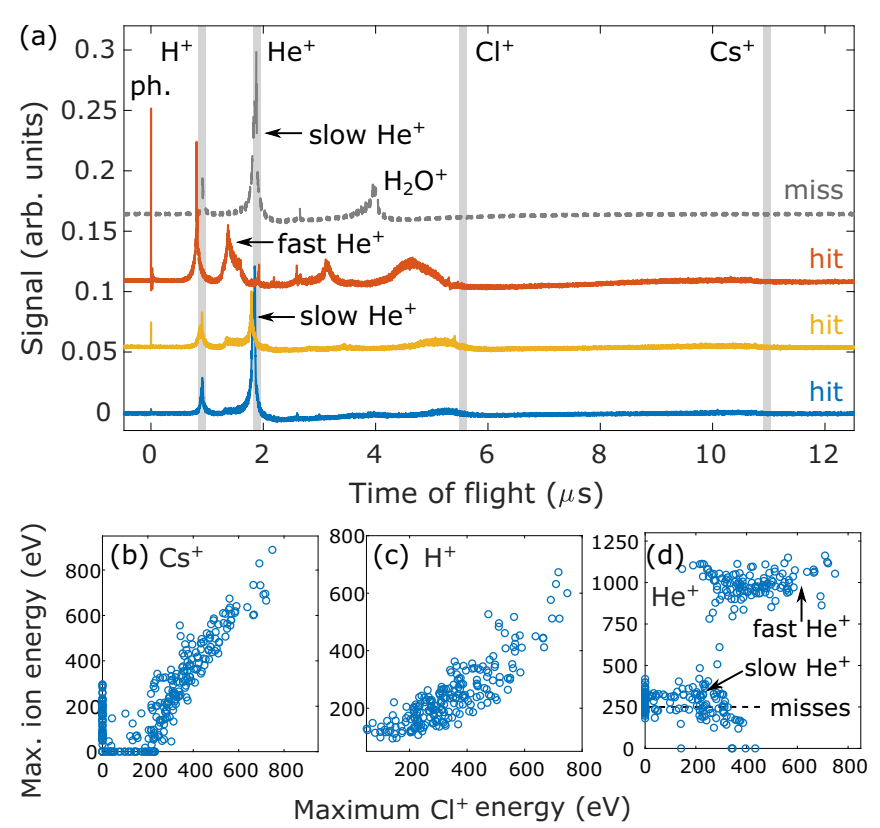

FIG. 1. (a) Representative single-shot ion ToF traces of three different hits of a laser-irradiated $\mathrm{CsCl}$ nanoparticle in gas environment (blue, yellow, and red lines, labeled "hit") and a single-shot trace of only the laser-irradiated gas (top dashed gray line, labeled "miss"). Traces are vertically offset for clarity. Ph. stands for photon peak. ToF positions of ions with zero kinetic energy are indicated by gray vertical lines. (b)-(d) Maximum energy of individual ions versus maximum energy of $\mathrm{Cl}^{+}$ions for single-shot hits: (b) $\mathrm{Cs}^{+}$ions, (c) $\mathrm{H}^{+}$ions, and (d) $\mathrm{He}^{+}$ions. Black dashed line at $250 \mathrm{eV}$ in (d) is an averaged value of maximum $\mathrm{He}^{+}$energy as extracted from an analysis of 120 traces of misses.

a number density in the interaction region of $\sim 5 \times 10^{15} \mathrm{~cm}^{-3}$ [30]. Note that the combination of a GDVN and ALS creates a target that consists of different atomic species inside the nanoparticle and in the surrounding gas.

The nanoparticle beam was irradiated by a $1-\mathrm{kHz} 800-\mathrm{nm}$ laser with a pulse duration of $120 \mathrm{fs}$ (FWHM) and a peak intensity of $2 \times 10^{15} \mathrm{~W} \mathrm{~cm}^{-2}$. The focal spot diameter was around $25 \mu \mathrm{m}$. Ions or electrons after the interaction were detected with the same microchannel plate detector placed in the direction perpendicular to both the laser and the nanoparticle beams. Electrons were detected in the drift mode. To estimate the electron kinetic energy a retarding voltage was applied to the drift tube. Ions were accelerated by planar electrodes providing a homogeneous electric field of $300 \mathrm{~V} \mathrm{~mm}^{-1}$. The laser polarization was towards the ion or electron detector. Single-shot data were acquired and, subsequently, sorted for hits and misses of the nanoparticle. Hits were identified by the appearance of a photon peak that originates from UV and $\mathrm{x}$-ray photons formed in the lasernanoparticle interaction [4,38]. The photon peak is not present when only gas interacts with the laser. The hit rate was around $25 \%$.

Single-shot ion ToF spectra of laser-irradiated $\mathrm{CsCl}$ nanoparticles in He gas environment are shown in Fig. 1(a). Ions with initial zero kinetic energy are expected to form a peak at ToFs indicated by the gray vertical lines. The observed shorter ToFs of individual ion peaks indicate that ions have a

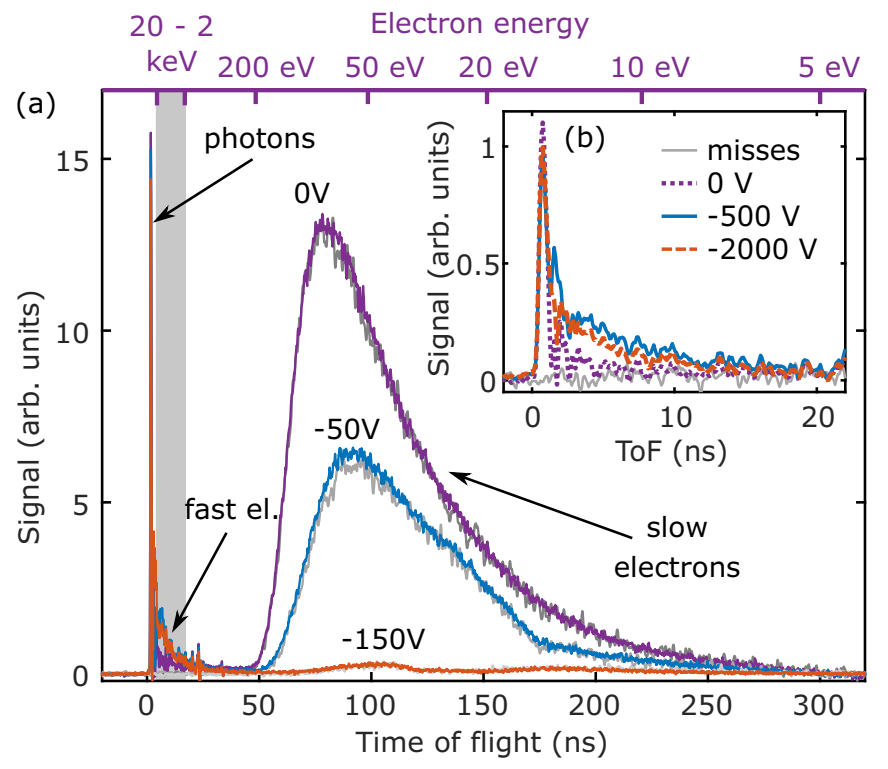

FIG. 2. Measured electron ToF traces of $\mathrm{CsCl}$ nanoparticles and the surrounding gas irradiated by a strong NIR laser pulse (peak intensity $2 \times 10^{15} \mathrm{~W} \mathrm{~cm}^{-2}$ ). (a) Light gray lines: averaged ToF traces of the surrounding gas (misses). Color (dark gray) lines: averaged ToF traces of nanoparticle hits. Retardation voltages applied to the drift tube are indicated next to each line. Fast and slow electron populations are indicated. The top axis indicates the expected electron energies for the retardation voltage of $0 \mathrm{~V}$. (b) Measured signal at short ToFs (fast electrons). Light gray line: averaged ToF trace of misses. Color (dark gray) lines: averaged ToF traces of nanoparticle hits for three different retardation voltages.

nonzero initial kinetic energy. For determination of ion's maximum kinetic energy we analyze the shortest ToF of each peak that corresponds to fastest ions with initial direction towards the detector. We theoretically calculate the ToF for each ion as a function its initial kinetic energy and take the kinetic energy that matches the experimental shortest ToF. Note the shot-toshot fluctuations in the ion spectra of hits. This is attributed to the different positions of the nanoparticle in the focal volume and to a possibility of hitting more than one nanoparticle. Our observation is in line with the findings of Refs. [39-41] where ion ToF spectra of single nanoparticles were sorted according to simultaneously measured coherent diffractive images. Figure 2 shows electron ToF traces measured under the same conditions as the ion traces in Fig. 1(a). The top axis indicates expected electron energies determined from their ToF for a retardation voltage of $0 \mathrm{~V}$. Electron $\mathrm{ToF}$ traces were first sorted for hits and misses and then averaged over about 1000 single-shot measurements. For nanoparticle hits, fast electrons with energies in the keV range are observed [Fig. 2(b)].

The main observation of our Letter is presented in Figs. 1(b)-1(d). Although single-shot maximum kinetic energies of $\mathrm{Cs}^{+}, \mathrm{H}^{+}$, and $\mathrm{Cl}^{+}$ions show mutual correlations [Figs. 1(b) and 1(c)], maximum kinetic energies of $\mathrm{He}^{+}$ions split into two fractions: slow (with maximum kinetic energy of around $250 \mathrm{eV}$ ) and fast (with maximum kinetic energy of around $1 \mathrm{keV}$ ) [Fig. 1(d)]. $\mathrm{He}^{+}$maximum energies are not correlated to the kinetic energies of $\mathrm{Cl}^{+}, \mathrm{Cs}^{+}$, and $\mathrm{H}^{+}$fragments. Note, that the maximum kinetic energy of the fast $\mathrm{He}^{+}$ 
fraction of $\sim 1 \mathrm{keV}$ is higher than the maximum kinetic energy of the $\mathrm{Cl}^{+}, \mathrm{Cs}^{+}$, and $\mathrm{H}^{+}$fragments. This is surprising because $\mathrm{Cs}^{+}, \mathrm{Cl}^{+}$, (and $\mathrm{H}^{+}$coming from water in the nanoparticle) ions originate from the nanoparticle with a solid density of around $10^{22} \mathrm{~cm}^{-3}$ (overdense plasma), whereas $\mathrm{He}^{+}$ions arise from the background gas with a substantially lower density of $\sim 5 \times 10^{15} \mathrm{~cm}^{-3}$ (extremely underdense plasma with $n_{e} / n_{c}=3 \times 10^{-6}$, where $n_{c}=1.7 \times$ $10^{21} \mathrm{~cm}^{-3}$ is the critical density for 800 -nm light).

In electron ToF traces of hits two electron fractions are observed: slow electrons with kinetic energies up to $200 \mathrm{eV}$ (and a mean energy of $60 \mathrm{eV}$ ) and fast electrons with energies in the keV range (Fig. 2). The slow electron signal contains contributions both from the nanoparticle and the surrounding gas, which cannot be separated in this measurement. To characterize the energies of the electron populations we apply a retardation voltage to the drift tube of the electron ToF spectrometer. The signal from the slow electron population disappears almost entirely when a retardation voltage above $150 \mathrm{~V}$ is used. The fast electron signal, originating only from the nanoparticle, first increases with the applied retardation voltage due to focusing of the electron trajectories onto the detector and then decreases because electrons do not arrive at the detector [Fig. 2(b)]. This confirms that the signal at short flight times $(\sim 1.5-20 \mathrm{~ns})$ can be attributed to electrons and not only to photons. From the signal measured with the highest applied retardation voltage of $-2 \mathrm{kV}$ and from the electrons' ToFs we estimate the energy of the fast electrons to be in the range of $2-20 \mathrm{keV}$. The large uncertainty in the determination of the fast electron energies is due to their very short flight times and to the limited range of retardation voltages that could be applied.

In the following, we discuss: (i) the laser interaction with the background He gas as it is revealed from ion and electron signal of misses, (ii) the dynamics of the nanoparticle explosion based on the observation of accelerated $\mathrm{Cs}^{+}, \mathrm{Cl}^{+}$, and $\mathrm{H}^{+}$ions and the slow population of electrons. Finally, we turn to (iii) the mutual interaction of the exploding nanoparticle and the He gas environment where a distinct nanoparticleassisted acceleration mechanism of $\mathrm{He}^{+}$is revealed.

(i) The interaction dynamics of intense laser pulses with the background $\mathrm{He}$ gas can be understood from the single-shot ion ToF traces of misses [Fig. 1(a), light gray dashed line], which look very similar for each shot. All ToF traces of misses contain moderately accelerated $\mathrm{He}^{+}$peak as revealed by a slight broadening and a shift of this peak towards shorter ToF. Additionally, a small $\mathrm{H}^{+}$and background $\mathrm{H}_{2} \mathrm{O}^{+}$peaks are observed. The estimated maximum kinetic energy of $\mathrm{He}^{+}$ions is around $250 \mathrm{eV}$. The appearance of this slow $\mathrm{He}^{+}$signal is well understood from our previous study [30]. Helium gas is ionized at the leading edge of the laser pulse. The laser field drives electrons from the laser axis by the ponderomotive force, and a positively charged $\mathrm{He}^{+}$plasma channel is created. This channel explodes due to Coulomb repulsion forces, leading to acceleration of $\mathrm{He}^{+}$ions. For our conditions we expect $\mathrm{He}^{+}$maximum energy to be $\sim 200-400 \mathrm{eV}$ [30], which agrees with the experimentally determined averaged value of $\sim 250 \mathrm{eV}$ extracted from an analysis of 120 single traces of misses [Fig. 1(d)]. In electron spectra of misses (Fig. 2, light gray lines) electrons with maximum kinetic energy of below
$200 \mathrm{eV}$ are identified, which corresponds to the above threshold ionization cutoff expected for our laser intensity [42].

(ii) Single-shot ion ToF traces of hits contain accelerated ions from the nanoparticle $\left(\mathrm{Cs}^{+}, \mathrm{Cl}^{+}\right.$, and $\left.\mathrm{H}^{+}\right)$with maximum kinetic energies of several $100 \mathrm{eV}$. The maximum kinetic energies of $\mathrm{Cs}^{+}, \mathrm{Cl}^{+}$, and $\mathrm{H}^{+}$ions show mutual correlations [Figs. 1(b) and 1(c)], indicating a common acceleration mechanism of these species. The acceleration mechanism of nanoparticle ions is well understood in terms of a hydrodynamic expansion of a nanoplasma $[8,31,43]$. Under intense irradiation conditions atoms inside the nanoparticle are ionized by tunnel ionization. A small fraction of created electrons (typically around $1 \%$ ) can escape the nanoparticle and leave a positive charge behind. Most of the activated electrons are trapped by the Coulomb potential and build a quasineutral nanoplasma inside the nanoparticle. Trapped electrons drive the nanoparticle expansion due to hydrodynamic pressure.

We have modeled the nanoplasma dynamics by the model of Ditmire et al. [31]. In the calculation we used an Ar nanoparticle with a diameter of $84 \mathrm{~nm}$ exposed to a $800-\mathrm{nm}$ pulse with 120-fs FWHM pulse duration and a peak intensity of up to $10^{16} \mathrm{~W} \mathrm{~cm}^{-2}$. The hydrodynamic model cannot include the two atomic species $(\mathrm{Cs}$ and $\mathrm{Cl})$ in the nanoparticle, but Ar serves as a good model system with similar mass and ionization energy as $\mathrm{Cl}$. In the nanoplasma model, the nanoparticle is treated as a uniform sphere, and electron ionization, heating, and nanoparticle expansion are described by rate equations of the corresponding quantities. The calculated maximum ion energy is $700 \mathrm{eV}$, in agreement with measured $\mathrm{Cl}^{+}$and $\mathrm{Cs}^{+}$energies [Fig. 1(b)]. The predicted electron energy is around $100 \mathrm{eV}$, which corresponds to the measured energies of the slow electron population [Fig. 2(a)]. These slow electrons, trapped inside the nanoparticle, drive the hydrodynamic expansion of the nanoparticle with a characteristic speed being the plasma sound speed: $\sqrt{Z k_{B} T_{e} / m_{i}}$. For our case, ion charge $Z=1$, electron temperature $k_{B} T_{e}=70 \mathrm{eV}$ and ion mass (for chlorine) $m_{i}=35.45 \mathrm{amu}$, the plasma sound speed is $1.4 \times 10^{4} \mathrm{~ms}^{-1}$. This leads to a characteristic expansion time of $\sim 7 \mathrm{ps}$ in which the nanoparticle doubles its radius. The explosion of $\mathrm{Cs}$ ions is even slower and should not affect the He acceleration, which takes place on a shorter timescale.

(iii) We now turn to the combined explosion of the nanoparticle and the surrounding gas. How does the highly ionized nanoparticle affect the surrounding $\mathrm{He}^{+}$ions? As is known from laser-driven ion acceleration experiments [23-29], $\mathrm{He}^{+}$ions cannot be directly accelerated by the laser field. Instead, they are accelerated by the electric field of the nanoparticle. According to general understanding, fast electrons, which leave the target very quickly, are not involved in the dynamics of the nanoparticle expansion, and their contribution is commonly not considered [24]. We suppose that fast electrons from the nanoparticle are created by a rescattering process $[9,38,44,45]$ on a timescale of the laser pulse duration (120 fs) and escape from the nanoparticle long before the expansion process sets in (e.g., 5-keV electrons travel $13 \mu \mathrm{m}$ in around $300 \mathrm{fs}$ ). We suggest that the fast electron population creates a charge on the nanoparticle that can lead to an acceleration of the surrounding $\mathrm{He}^{+}$to kinetic energies of $\sim 1 \mathrm{keV}$. 

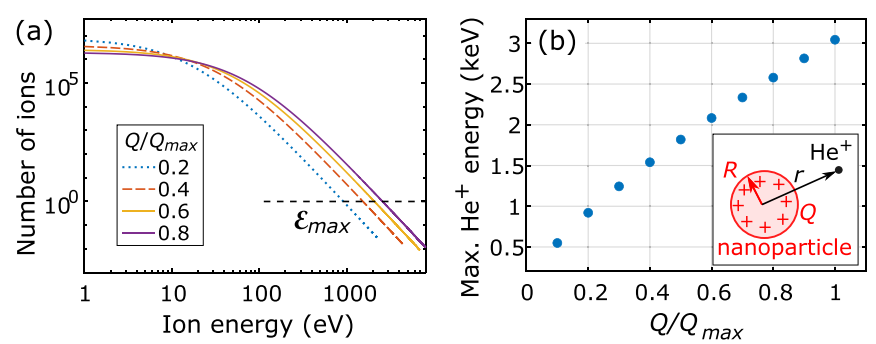

FIG. 3. (a) Calculated $\mathrm{He}^{+}$energy spectra for $R_{\max }=$ $13 \mu \mathrm{m}, Q_{\max }=8 \times 10^{5} e$ and different values of charge $Q / Q_{\max }$. Maximum $\mathrm{He}^{+}$energy $\mathcal{E}_{\text {max }}$ is indicated. (b) Calculated maximum $\mathrm{He}^{+}$energy as a function of $Q / Q_{\max }$. Other parameters are the same as in (a). The inset shows a schematic geometry used for the calculation.

In order to evaluate the energies that $\mathrm{He}^{+}$ions can gain in the field created on the nanoparticle after the fast electrons have left, we assume a simplified stationary case where fast electrons have already expanded to a few micrometer distance from the nanoparticle, but the nanoparticle expansion has not yet started. We consider a nanoparticle with radius $R$, a positive charge $Q$ on its surface, and a quasineutral core [inset in Fig. 3(b)]. We show that ions accelerated by the nanoparticle field can gain energies in the keV range. The stationary model applied here is valid for the first few picoseconds after the laser pulse before the nanoparticle expands and the positively charged shell at the nanoparticle surface is destroyed.

The charge $Q$, determined by the number of fast electrons that have left the nanoparticle, is estimated by considering suppression of the nanoparticle electric field by the laser field $E_{\text {laser }}$ [46]. The maximum charge $Q_{\max }$ that can be built on the nanoparticle by this process is $Q_{\max }=4 \pi \varepsilon_{0} R^{2} E_{\text {laser }}$, where $\varepsilon_{0}$ is the vacuum permittivity. For $R=100 \mathrm{~nm}$ and laser intensity of $2 \times 10^{15} \mathrm{~W} \mathrm{~cm}^{-2}$ we get $Q_{\max }=8 \times 10^{5} e$, where $e$ is the electron charge. For the following calculations we consider $Q / Q_{\max }<1$ in order to take into account partial suppression of the potential barrier and a lower local laser intensity at the position of the nanoparticle.

Considering a spherical nanoparticle with charge $Q$, the electric field at a distance $r$ from the nanoparticle center is $E(r)=Q /\left(4 \pi \varepsilon_{0} r^{2}\right) . \mathrm{He}^{+}$ion with charge $\mathrm{Ze}(Z=1)$ at position $r$ is accelerated by this field to energy,

$$
\mathcal{E}(r)=Z e \int_{r}^{R_{\max }} E\left(r^{\prime}\right) d r^{\prime}=\frac{Q Z e}{4 \pi \varepsilon_{0}}\left(\frac{1}{r}-\frac{1}{R_{\max }}\right),
$$

where the upper limit for the integration $R_{\max }$ is taken to be the focal spot radius $\left(R_{\max }=13 \mu \mathrm{m}\right)$. As will be shown later, the exact choice of $R_{\max }$ does not affect the main outcome of the calculation.

Considering the number $d N$ of ions in a shell with radius $r$ and width $d r$ to be $d N=4 \pi r^{2} n d r$ (where $n$ is the helium number density), we obtain the ion energy spectrum,

$$
\frac{d N}{d \mathcal{E}}=\frac{16 \pi^{2} \varepsilon_{0} n}{Q Z e} \frac{1}{\left(\frac{1}{R_{\max }}+\frac{4 \pi \varepsilon_{0}}{Q Z e} \mathcal{E}\right)^{4}} .
$$

Calculated $\mathrm{He}^{+}$spectra are shown in Fig. 3(a). The maximum $\mathrm{He}^{+}$energy for each spectrum is taken as a value for which the number of $\mathrm{He}^{+}$ions falls below 1 , indicated by a dashed line in Fig. 3(a). This is a more reasonable value than the absolute maximum energy evaluated from Eq. (1) for $r=R$ (i.e., for a $\mathrm{He}^{+}$ion initially located at the nanoparticle surface). The maximum $\mathrm{He}^{+}$energies [Fig. 3(b)] are on the order of $1 \mathrm{keV}$, in a good agreement with the experiment.

The calculated $\mathrm{He}^{+}$energy spectra [Eq. (2)] depend mainly on the charge $Q$. This is because charge $Q$, determined by the number of electrons that have left the nanoparticle, defines the strength of the electric field that accelerates $\mathrm{He}^{+}$ions. Nevertheless, for different values of $Q$ the maximum $\mathrm{He}^{+}$ energy is on the order of $1 \mathrm{keV}$. The term $1 / R_{\max }$ in Eqs. (1) and (2) is considerably smaller than other terms, thus, the specific choice of $R_{\max }$ does not change the calculation outcome. The spectra do not depend on the nanoparticle radius $R$ because of the chosen definition of the maximum energy. Therefore, an uncertainty in $R$ and a probable distribution of nanoparticle sizes do not affect the calculated $\mathrm{He}^{+}$energies. Accordingly, our calculation explains well the observed $\mathrm{He}^{+}$ energies with a limited number of input parameters. For a more accurate calculation of $\mathrm{He}^{+}$acceleration in the presence of the nanoparticle, a detailed numerical model containing dynamics and collective effects is needed.

In conclusion, we have demonstrated an efficient acceleration of low-density $\mathrm{He}^{+}$ions to $\sim 1-\mathrm{keV}$ energies with medium laser intensities. The critical role in this process is played by the nanoparticle. We suggest that the accumulated positive charge on the nanoparticle surface creates an electric field that accelerates $\mathrm{He}^{+}$ions in the surrounding gas. The maximum kinetic energy of the $\mathrm{He}^{+}$ions gained through this interaction is higher than the maximum kinetic energies of $\mathrm{Cs}^{+}, \mathrm{Cl}^{+}$, and $\mathrm{H}^{+}$ions, which are driven mainly by the hydrodynamic explosion of the nanoparticle. The acceleration mechanism presented here is not restricted to the interactions with a strong NIR field because highly ionized nanoparticles can also be produced by intense x rays [39-41]. For example, detecting ions (together with plasma emission) from the interaction of nanoparticles with intense $\mathrm{x}$-ray pulses is a promising application for on-line data reduction at high-repetition rate experiments at free electron lasers [41,47]. Therefore, the acceleration mechanism shown here should be of high relevance for large number of systems, including rare-gas clusters, (bio) molecules in a gas environment, and nanotargets prepared by the laser ablation, irradiated by intense lasers within a broad photon-energy range from NIR to $x$ rays.

We acknowledge technical support from D. Westphal (Uppsala University), O. Janda, Z. Svoboda, and A. Wolf. We acknowledge ELI Beamlines in Dolní Břežany, Czech Republic for providing beam time and thank the instrument group and facility staff for their assistance. LM2018141, MEYSlarge research infrastructure project ELI Beamlines. This work was supported by the Projects Advanced research using high intensity laser produced photons and particles (ADONIS) (Grant No. CZ.02.1.01/0.0/0.0/16_019/0000789) and Structural dynamics of biomolecular systems (ELIBIO) (Grant No. CZ.02.1.01/0.0/0.0/15_003/0000447), both from the European Regional Development Fund, and the Ministry of Education, Youth and Sports. 
[1] E. M. Snyder, S. A. Buzza, and A. W. Castleman, Jr., Intense Field-Matter Interactions: Multiple Ionization of Clusters, Phys. Rev. Lett. 77, 3347 (1996).

[2] T. Ditmire, J. W. G. Tisch, E. Springate, M. B. Mason, N. Hay, R. A. Smith, J. Marangos, and M. H. R. Hutchinson, High-energy ions produced in explosions of superheated atomic clusters, Nature (London) 386, 54 (1997).

[3] Y. Fukuda, A. Y. Faenov, M. Tampo, T. A. Pikuz, T. Nakamura, M. Kando, Y. Hayashi, A. Yogo, H. Sakaki, T. Kameshima, A. S. Pirozhkov, K. Ogura, M. Mori, T. Z. Esirkepov, J. Koga, A. S. Boldarev, V. A. Gasilov, A. I. Magunov, T. Yamauchi, R. Kodama, P. R. Bolton, Y. Kato, T. Tajima, H. Daido, and S. V. Bulanov, Energy Increase in Multi-MeV Ion Acceleration in the Interaction of a Short Pulse Laser with a Cluster-Gas Target, Phys. Rev. Lett. 103, 165002 (2009).

[4] E. Springate, S. A. Aseyev, S. Zamith, and M. J. J. Vrakking, Electron kinetic energy measurements from laser irradiation of clusters, Phys. Rev. A 68, 053201 (2003).

[5] Y. Fukuda, Y. Akahane, M. Aoyama, Y. Hayashi, T. Homma, N. Inoue, M. Kando, S. Kanazawa, H. Kiriyama, S. Kondo et al., Ultrarelativistic electron generation during the intense, ultrashort laser pulse interaction with clusters, Phys. Lett. A 363, 130 (2007).

[6] A. McPherson, B. D. Thompson, A. B. Borisov, K. Boyer, and C. K. Rhodes, Multiphoton-induced X-ray emission at 4-5 keV from $\mathrm{Xe}$ atoms with multiple core vacancies, Nature (London) 370, 631 (1994).

[7] S. G. Bochkarev, A. Faenov, T. Pikuz, A. V. Brantov, V. F. Kovalev, I. Skobelev, S. Pikuz, R. Kodama, K. I. Popov, and V. Y. Bychenkov, Ion energy spectra directly measured in the interaction volume of intense laser pulses with clustered plasma, Sci. Rep. 8, 9404 (2018).

[8] D. D. Hickstein, F. Dollar, J. A. Gaffney, M. E. Foord, G. M. Petrov, B. B. Palm, K. E. Keister, J. L. Ellis, C. Ding, S. B. Libby, J. L. Jimenez, H. C. Kapteyn, M. M. Murnane, and W. Xiong, Observation and Control of Shock Waves in Individual Nanoplasmas, Phys. Rev. Lett. 112, 115004 (2014).

[9] S. Zherebtsov, T. Fennel, J. Plenge, E. Antonsson, I. Znakovskaya, A. Wirth, O. Herrwerth, F. Suessmann, C. Peltz, I. Ahmad et al., Controlled near-field enhanced electron acceleration from dielectric nanospheres with intense few-cycle laser fields, Nat. Phys. 7, 656 (2011).

[10] F. Suessmann, L. Seiffert, S. Zherebtsov, V. Mondes, J. Stierle, M. Arbeiter, J. Plenge, P. Rupp, C. Peltz, A. Kessel et al., Field propagation-induced directionality of carrier-envelope phasecontrolled photoemission from nanospheres, Nat. Commun. 6, 7944 (2015).

[11] Q. Liu, S. Zherebtsov, L. Seiffert, S. Skruszewicz, D. Zietlow, S. Ahn, P. Rupp, P. Wnuk, S. Sun, A. Kessel et al., Alloptical spatio-temporal control of electron emission from $\mathrm{SiO}_{2}$ nanospheres with femtosecond two-color laser fields, New J. Phys. 21, 073011 (2019).

[12] D. D. Hickstein, F. Dollar, J. L. Ellis, K. J. Schnitzenbaumer, K. E. Keister, G. M. Petrov, C. Ding, B. B. Palm, J. A. Gaffney, M. E. Foord et al., Mapping nanoscale absorption of femtosecond laser pulses using plasma explosion imaging, ACS Nano 8 , 8810 (2014).

[13] J. Passig, S. Zherebtsov, R. Irsig, M. Arbeiter, C. Peltz, S. Goede, S. Skruszewicz, K.-H. Meiwes-Broer, J. Tiggesbaeumker, M. F. Kling, and T. Fennel, Nanoplasmonic electron acceleration by attosecond-controlled forward rescattering in silver clusters, Nat. Commun. 8, 1181 (2017).

[14] H. Ruf, C. Handschin, R. Cireasa, N. Thiré, A. Ferré, S. Petit, D. Descamps, E. Mével, E. Constant, V. Blanchet, B. Fabre, and Y. Mairesse, Inhomogeneous High Harmonic Generation in Krypton Clusters, Phys. Rev. Lett. 110, 083902 (2013).

[15] R. A. Ganeev, C. Hutchison, T. Witting, F. Frank, S. Weber, W. A. Okell, E. Fiordilino, D. Cricchio, F. Persico, A. Zaïr, J. W. G. Tisch, and J. P. Marangos, High-order harmonic generation in fullerenes using few- and multi-cycle pulses of different wavelengths, J. Opt. Soc. Am. B 30, 7 (2013).

[16] B. Bódi, M. Aladi, P. Rácz, I. B. Földes, and P. Dombi, High harmonic generation on noble gas clusters, Opt. Express 27, 26721 (2019).

[17] M. H. Cho, V. B. Pathak, H. T. Kim, and C. H. Nam, Controlled electron injection facilitated by nanoparticles for laser wakefield acceleration, Sci. Rep. 8, 16924 (2018).

[18] C. Aniculaesei, V. B. Pathak, K. H. Oh, P. K. Singh, B. R. Lee, C. I. Hojbota, T. G. Pak, E. Brunetti, B. J. Yoo, J. H. Sung, S. K. Lee, H. T. Kim, and C. H. Nam, Proof-of-Principle Experiment for Nanoparticle-Assisted Laser Wakefield Electron Acceleration, Phys. Rev. Appl. 12, 044041 (2019).

[19] M. Tchaplyguine, R. R. Marinho, M. Gisselbrecht, J. Schulz, N. Mårtensson, S. L. Sorensen, A. Naves de Brito, R. Feifel, G. Öhrwall, M. Lundwall et al., The size of neutral free clusters as manifested in the relative bulk-to-surface intensity in core level photoelectron spectroscopy, J. Chem. Phys. 120, 345 (2004).

[20] D. Rolles, H. Zhang, Z. Pešć, J. Bozek, and N. Berrah, Emergence of valence band structure in rare-gas clusters, Chem. Phys. Lett. 468, 148 (2009).

[21] T. Oelze, B. Schütte, J. P. Müller, M. Müller, M. Wieland, U. Frühling, M. Drescher, T. Golz, A. Al-Shemmary, N. Stojanovic, and M. Krikunova, Mapping ultrafast ionization of atoms and clusters with terahertz-streaking delay, Phys. Rev. A 99, 043423 (2019).

[22] M. F. Ciappina, T. Shaaran, R. Guichard, J. A. PérezHernández, L. Roso, M. Arnold, T. Siegel, A. Zaïr, and M. Lewenstein, High energy photoelectron emission from gases using plasmonic enhanced near-fields, Laser Phys. Lett. 10, 105302 (2013).

[23] R. A. Snavely, M. H. Key, S. P. Hatchett, T. E. Cowan, M. Roth, T. W. Phillips, M. A. Stoyer, E. A. Henry, T. C. Sangster, M. S. Singh, S. C. Wilks, A. MacKinnon, A. Offenberger, D. M. Pennington, K. Yasuike, A. B. Langdon, B. F. Lasinski, J. Johnson, M. D. Perry, and E. M. Campbell, Intense HighEnergy Proton Beams From Petawatt-Laser Irradiation of Solids, Phys. Rev. Lett. 85, 2945 (2000).

[24] S. P. Hatchett, C. G. Brown, T. E. Cowan, E. A. Henry, J. S. Johnson, M. H. Key, J. A. Koch, A. B. Langdon, B. F. Lasinski, R. W. Lee et al., Electron, photon, and ion beams from the relativistic interaction of Petawatt laser pulses with solid targets, Phys. Plasmas 7, 2076 (2000).

[25] A. Macchi, M. Borghesi, and M. Passoni, Ion acceleration by superintense laser-plasma interaction, Rev. Mod. Phys. 85, 751 (2013).

[26] S. Zhao, C. Lin, H. Y. Wang, H. Y. Lu, X. T. He, J. E. Chen, T. E. Cowan, and X. Q. Yan, Ion acceleration enhanced by target ablation, Phys. Plasmas 22, 073106 (2015).

[27] Q. Liao, M. J. Wu, Z. Gong, Y. X. Geng, X. H. Xu, D. Y. Li, Y. R. Shou, J. G. Zhu, C. C. Li, M. Yang et al., Enhanced laser 
proton acceleration by target ablation on a femtosecond laser system, Phys. Plasmas 25, 063109 (2018).

[28] D. Margarone, O. Klimo, I. J. Kim, J. Prokůpek, J. Limpouch, T. M. Jeong, T. Mocek, J. Pšikal, H. T. Kim, J. Proška, K. H. Nam, L. Štolcová, I. W. Choi, S. K. Lee, J. H. Sung, T. J. Yu, and G. Korn, Laser-Driven Proton Acceleration Enhancement by Nanostructured Foils, Phys. Rev. Lett. 109, 234801 (2012).

[29] D. Levy, C. Bernert, M. Rehwald, I. A. Andriyash, S. Assenbaum, T. Kluge, E. Kroupp, L. Obst-Huebl, R. Pausch, A. Schulze-Makuch et al., Laser-plasma proton acceleration with a combined gas-foil target, New J. Phys. 22, 103068 (2020).

[30] E. Klimešová, O. Kulyk, Y. Gu, L. Dittrich, G. Korn, J. Hajdu, M. Krikunova, and J. Andreasson, Plasma channel formation in NIR laser-irradiated carrier gas from an aerosol nanoparticle injector, Sci. Rep. 9, 8851 (2019).

[31] T. Ditmire, T. Donnelly, A. M. Rubenchik, R. W. Falcone, and M. D. Perry, Interaction of intense laser pulses with atomic clusters, Phys. Rev. A 53, 3379 (1996).

[32] E. Klimešová, O. Kulyk, Z. Hoque, A. H. Roos, K. P. Khakurel, M. Rebarz, M. Jurkovič, M. Albrecht, O. Finke, R. Lera et al., A multipurpose end-station for atomic, molecular and optical sciences and coherent diffractive imaging at ELI Beamlines, Eur. Phys. J.: Spec. Top. (2021), doi:10.1140/epjs/s11734-02100192-z.

[33] M. F. Hantke, J. Bielecki, O. Kulyk, D. Westphal, D. S. D. Larsson, M. Svenda, H. K. N. Reddy, R. A. Kirian, J. Andreasson, J. Hajdu, and F. R. N. C. Maia, Rayleigh-scattering microscopy for tracking and sizing nanoparticles in focused aerosol beams, IUCrJ 5, 673 (2018).

[34] E. Sobolev, S. Zolotarev, K. Giewekemeyer, J. Bielecki, K. Okamoto, H. K. N. Reddy, J. Andreasson, K. Ayyer, I. Barak, S. Bari et al., Megahertz single-particle imaging at the European XFEL, Commun. Phys. 3, 97 (2020).

[35] D. P. DePonte, U. Weierstall, K. Schmidt, J. Warner, D. Starodub, J. C. H. Spence, and R. B. Doak, Gas dynamic virtual nozzle for generation of microscopic droplet streams, J. Phys. D: Appl. Phys. 41, 195505 (2008)

[36] N. Roth, S. Awel, D. A. Horke, and J. Küpper, Optimizing aerodynamic lenses for single-particle imaging, J. Aerosol Sci. 124, 17 (2018).

[37] G. E. Ewing and S. J. Peters, Adsorption of water on NaCl, Surf. Rev. Lett. 04, 757 (1997).

[38] B. Schuette, P. Ye, S. Patchkovskii, D. R. Austin, C. Brahms, C. Strueber, T. Witting, M. Y. Ivanov, J. W. G. Tisch, and J. P. Marangos, Strong-field ionization of clusters using two-cycle pulses at $1.8 \mu \mathrm{m}$, Sci. Rep. 6, 39664 (2016).
[39] T. Gorkhover, M. Adolph, D. Rupp, S. Schorb, S. W. Epp, B. Erk, L. Foucar, R. Hartmann, N. Kimmel, K.-U. Kühnel, D. Rolles, B. Rudek, A. Rudenko, R. Andritschke, A. Aquila, J. D. Bozek, N. Coppola, T. Erke, F. Filsinger, H. Gorke, H. Graafsma, L. Gumprecht, G. Hauser, S. Herrmann, H. Hirsemann, A. Hömke, P. Holl, C. Kaiser, F. Krasniqi, J.H. Meyer, M. Matysek, M. Messerschmidt, D. Miessner, B. Nilsson, D. Pietschner, G. Potdevin, C. Reich, G. Schaller, C. Schmidt, F. Schopper, C. D. Schröter, J. Schulz, H. Soltau, G. Weidenspointner, I. Schlichting, L. Strüder, J. Ullrich, T. Möller, and C. Bostedt, Nanoplasma Dynamics of Single Large Xenon Clusters Irradiated with Superintense X-Ray Pulses from the Linac Coherent Light Source Free-Electron Laser, Phys. Rev. Lett. 108, 245005 (2012).

[40] D. Rupp, L. Flückiger, M. Adolph, T. Gorkhover, M. Krikunova, J. P. Müller, M. Müller, T. Oelze, Y. Ovcharenko, B. Röben, M. Sauppe, S. Schorb, D. Wolter, R. Mitzner, M. Wöstmann, S. Roling, M. Harmand, R. Treusch, M. Arbeiter, T. Fennel, C. Bostedt, and T. Möller, Recombination-Enhanced Surface Expansion of Clusters in Intense Soft X-Ray Laser Pulses, Phys. Rev. Lett. 117, 153401 (2016).

[41] J. Andreasson, A. V. Martin, M. Liang, N. Timneanu, A. Aquila, F. Wang, B. Iwan, M. Svenda, T. Ekeberg, M. Hantke et al., Automated identification and classification of single particle serial femtosecond X-ray diffraction data, Opt. Express 22, 2497 (2014).

[42] G. G. Paulus, W. Becker, W. Nicklich, and H. Walther, Rescattering effects in above-threshold ionization: A classical model, J. Phys. B: At., Mol. Opt. Phys. 27, L703 (1994).

[43] T. Fennel, K.-H. Meiwes-Broer, J. Tiggesbäumker, P.-G. Reinhard, P. M. Dinh, and E. Suraud, Laser-driven nonlinear cluster dynamics, Rev. Mod. Phys. 82, 1793 (2010).

[44] Z. Wang, A. Camacho Garibay, H. Park, U. Saalmann, P. Agostini, J. M. Rost, and L. F. DiMauro, Universal High-Energy Photoelectron Emission from Nanoclusters Beyond the Atomic Limit, Phys. Rev. Lett. 124, 173201 (2020).

[45] T. Taguchi, T. M. Antonsen, and H. M. Milchberg, Resonant Heating of a Cluster Plasma by Intense Laser Light, Phys. Rev. Lett. 92, 205003 (2004).

[46] U. Saalmann and J. M. Rost, Electron dynamics in strong laser pulse illumination of large rare gas clusters, Eur. Phys. J. D 36, 159 (2005).

[47] H. O. Jönsson, C. Caleman, J. Andreasson, and N. Tîmneanu, Hit detection in serial femtosecond crystallography using X-ray spectroscopy of plasma emission, IUCrJ 4, 778 (2017). 